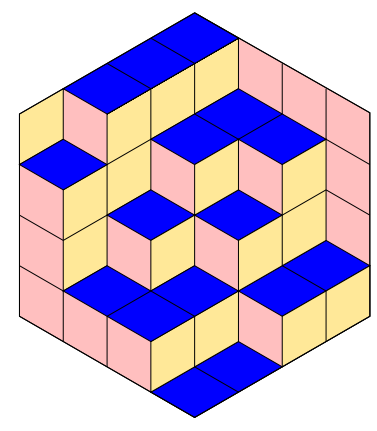

ALGEBRAIC COMBINATORICS

Edward Richmond \& Vasu Tewari

Noncommutative $L R$ coefficients and crystal reflection operators

Volume 4, issue 1 (2021), p. 145-162.

<http://alco.centre-mersenne.org/item/ALCO_2021__4_1_145_0>

(C) The journal and the authors, 2021.

Some rights reserved.

(c) BY This article is licensed under the

Creative Commons ATtribution 4.0 International LiCEnSE.

http://creativecommons.org/licenses/by/4.0/

Access to articles published by the journal Algebraic Combinatorics on the website http://alco.centre-mersenne.org/ implies agreement with the Terms of Use (http://alco.centre-mersenne.org/legal/).

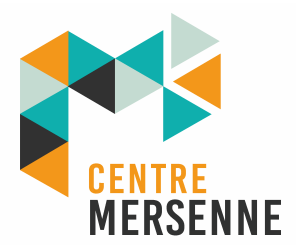

Algebraic Combinatorics is member of the Centre Mersenne for Open Scientific Publishing www.centre-mersenne.org 


\title{
Noncommutative LR coefficients and crystal reflection operators
}

\author{
Edward Richmond \& Vasu Tewari
}

\begin{abstract}
We relate noncommutative Littlewood-Richardson coefficients of BessenrodtLuoto-van Willigenburg to classical Littlewood-Richardson coefficients via crystal reflection operators. A key role is played by the combinatorics of frank words.
\end{abstract}

\section{INTRODUCTION}

Quasisymmetric Schur functions, introduced by Haglund-Luoto-Mason-van Willigenburg [14], form a prominent basis for the Hopf algebra of quasisymmetric functions QSym. The quasisymmetric Schur function indexed by the composition $\alpha$, denoted by $\mathcal{S}_{\alpha}$, is obtained by summing monomials attached to semistandard composition tableaux of shape $\alpha$. This is reminiscent of the definition of Schur functions as sums of monomials corresponding to semistandard Young tableaux. As the name suggests, quasisymmetric Schur functions share many properties with classical Schur functions, and Mason's map $\rho$ [24] connects the combinatorics of composition tableaux to that of Young tableaux. Understanding analogues of Schur functions and their generalizations has long been a theme in algebraic combinatorics; see [1, 2, 3, 4, 25, 32] for recent work in this context.

The Hopf algebra of noncommutative symmetric functions NSym, introduced in the seminal paper [12], is Hopf-dual to QSym as shown by Malvenuto-Reutenauer [23]. Bessenrodt-Luoto-van Willigenburg [5] studied the dual basis elements $\mathbf{s}_{\alpha}$ corresponding to quasisymmetric Schur functions. The resulting functions, also indexed by compositions, are called noncommutative Schur functions. The inclusion of the Hopf algebra of symmetric functions Sym into QSym induces a projection $\chi:$ NSym $\rightarrow$ Sym. This projection maps noncommutative Schur functions to classical Schur functions, and justifies the name of the former. The structure constants $C_{\alpha \beta}^{\gamma}$ that arise in

$$
\mathbf{s}_{\alpha} \cdot \mathbf{s}_{\beta}=\sum_{\gamma} C_{\alpha \beta}^{\gamma} \mathbf{s}_{\gamma}
$$

Manuscript received 19th August 2019, revised 11th August 2020, accepted 6th October 2020.

KEYwORDS. Crystal operator, Littlewood-Richardson coefficient, noncommutative symmetric function, symmetric function, Schur function. 
are called noncommutative Littlewood-Richardson (LR) coefficients. These coefficients turn out to be nonnegative integers that refine the classical LR coefficients $c_{\nu \mu}^{\lambda}$ that arise in the product of Schur functions

$$
s_{\nu} \cdot s_{\mu}=\sum_{\lambda} c_{\nu \mu}^{\lambda} s_{\lambda}
$$

More precisely, suppose $\nu$ and $\mu$ are partitions that rearrange to compositions $\alpha$ and $\beta$ respectively. Applying $\chi$ to both sides in (1) and comparing the result with (2) implies

$$
c_{\nu \mu}^{\lambda}=\sum_{\gamma} C_{\alpha \beta}^{\gamma}
$$

where the sum on the right runs over all compositions $\gamma$ that rearrange to $\lambda$. Among the numerous combinatorial interpretations of $c_{\nu \mu}^{\lambda}$, the one we focus on states that $c_{\nu \mu}^{\lambda}$ counts LR tableaux of shape $\lambda / \mu$ and content $\nu$. Our primary goal in this article is to describe the summands on the right hand side in (3) in terms of LR tableaux. To this end, crystal reflection operators are key.

To state our result, we introduce the necessary notation briefly. The reader is referred to Section 2 for details. Given a composition $\alpha$, we denote the partition underlying $\alpha$ by $\operatorname{sort}(\alpha)$. Let $\operatorname{LRT}(\lambda, \mu, \nu)$ be the set of LR tableaux of shape $\lambda / \mu$ and content $\nu$. Given a permutation $\sigma$, let $\operatorname{LRT}^{\sigma}(\lambda, \mu, \nu)$ be the set of tableaux obtained by applying the crystal reflection operators corresponding to a reduced word of $\sigma$. Let $\alpha:=\sigma \cdot \nu$, and let $\beta$ be any composition that satisfies $\operatorname{sort}(\beta)=\mu$. On applying the map $\rho_{\beta}^{-1}$ (which sends Young tableaux to composition tableaux, [22, Chapter 4]) to elements in $\operatorname{LRT}^{\sigma}(\lambda, \mu, \nu)$, we obtain the disjoint decomposition

$$
\operatorname{LRT}^{\sigma}(\lambda, \mu, \nu)=\coprod_{\gamma} X_{\alpha \beta}^{\gamma},
$$

where $X_{\alpha \beta}^{\gamma}$ consists of all tableaux $T \in \operatorname{LRT}^{\sigma}(\lambda, \mu, \nu)$ whose outer shape under $\rho_{\beta}^{-1}$ is given by the composition $\gamma$. Under this setup, our main theorem states the following.

THEOREM 1.1. The noncommutative LR coefficient $C_{\alpha \beta}^{\gamma}$ equals the cardinality of $X_{\alpha \beta}^{\gamma}$.

The upshot of Theorem 1.1 is that starting from $\operatorname{LRT}(\lambda, \mu, \nu)$, we can compute all noncommutative LR coefficients $C_{\alpha \beta}^{\gamma}$, where $\alpha$ dictates the choice of crystal reflections to be performed and $\beta$ determines the generalized $\rho$ map to be applied. We also interpret Theorem 1.1 in terms of chains in Young's lattice indexed by certain frank words and obtain a rule for $C_{\alpha \beta}^{\gamma}$ involving box-adding operators on compositions. In the case where $\alpha$ is either a partition or reverse partition, our interpretations yield the two LR rules in [6]. For an example demonstrating Theorem 1.1, see Subsection 3.2.

Note that LR coefficients can be interpreted as the number of lattice points in an appropriate convex polytope/cone [26]. Since Theorem 1.1 describes noncommutative LR coefficients by refining the set of LR tableaux, it is natural to ask if noncommutative LR coefficients count lattice points in some refinement of the corresponding polytope/cone. Data suggest that the analogue of the saturation conjecture, famously resolved by Knutson and Tao [15], holds for noncommutative LR coefficients as well. In fact (3) tells us that saturation of noncommutative LR coefficients implies that of the classical counterparts. This motivates studying these constants from a polytopal perspective, which is why the authors were interested in a statement like Theorem 1.1 in the first place.

OUthine OF THE ARTICLE. Section 2 sets up all the necessary combinatorial background. Section 3 describes our central result along with examples. Section 4.1 introduces growth words and frank words. Section 4.2 presents an outline of our proof strategy given its technical nature. Section 4.3 identifies LR tableaux with certain 
distinguished frank words called compatible words, drawing upon work by RemmelShimozono [29]. In Section 4.4, we describe the Lascoux-Schützenberger symmetric group action on frank words. Section 4.5 relates crystal reflections acting on LR tableaux to the aforementioned action restricted to compatible words. Section 5 relates the results in Section 4 back to noncommutative LR coefficients. We conclude our article with Corollary 5.4, which reinterprets our combinatorial interpretation for noncommutative LR coefficients in terms of box-adding operators on compositions.

\section{BACKGROUND}

To keep this section brief, we assume knowledge of combinatorial structures and algorithms arising in symmetric functions theory such as partitions, skew shapes, Young tableaux, Robinson-Schensted-Knuth insertion and jeu-de-taquin. The reader is referred to standard texts $[11,30,33]$ for further information.

Regarding notions relevant to the theory of quasisymmetric Schur functions, we adhere to the notation and conventions in [22]. We emphasize that what we call quasisymmetric Schur functions in this article are the Young quasisymmetric Schur functions in [22]. This choice, although cosmetic as both the functions are related via a simple involution, has its benefits. The tableau objects that we consider in the quasisymmetric/noncommutative setting align with the more prevalent notion of Young tableaux rather than reverse tableaux. Furthermore, it is the Young quasisymmetric Schurs that decompose the dual immaculate quasisymmetric functions [4] positively as shown recently in [2], thereby connecting two well-studied quasisymmetric analogues of Schurs.

2.1. Words. Throughout, we denote the set of positive integers by $\mathbb{Z}_{+}$. Given $n \in$ $\mathbb{Z}_{+}$, define $[n]:=\{1, \ldots, n\}$. Furthermore, set $[0]:=\varnothing$. Let $\mathbb{Z}_{+}^{*}$ denote the set of all words in the alphabet $\mathbb{Z}_{+}$.

Consider $w=w_{1} \cdots w_{n} \in \mathbb{Z}_{+}^{*}$. We call $n$ the length of $w$ and denote it by $|w|$. The word $w_{n} \cdots w_{1}$ is denoted by $w^{r}$. We say that $w$ is reverse-lattice if every suffix of $w$ contains at least as many $i$ 's as $i+1$ 's for all $i \in \mathbb{Z}_{+}$. Let $\mathfrak{S}_{n}$ be the permutation group on $[n]$ and denote the longest element in $\mathfrak{S}_{n}$ by $w_{0}^{(n)}$, or simply by $w_{0}$ if $n$ is clear from context. The permutation $\sigma \in \mathfrak{S}_{n}$ acts on a sequence $\lambda=\left(\lambda_{1}, \ldots, \lambda_{n}\right)$ by $\sigma \cdot \lambda=\left(\lambda_{\sigma^{-1}(1)}, \ldots, \lambda_{\sigma^{-1}(n)}\right)$. Note that this is a right action.

2.2. Compositions And PARTitions. A finite list of nonnegative integers $\alpha=$ $\left(\alpha_{1}, \ldots, \alpha_{\ell}\right)$ is called a weak composition. If $\alpha_{i}>0$ for all $1 \leqslant i \leqslant \ell$, then $\alpha$ is called a composition. If, in addition, we have $\alpha_{1} \geqslant \cdots \geqslant \alpha_{\ell}>0$, then $\alpha$ is called a partition. Given $\alpha=\left(\alpha_{1}, \ldots, \alpha_{\ell}\right)$ we call the $\alpha_{i}$ the parts of $\alpha$ and the sum of the $\alpha_{i}$, denoted by $|\alpha|$, is called the size of $\alpha$. We denote the number of parts of $\alpha$ by $\ell(\alpha)$ and call it the length of $\alpha$. The unique composition of length and size zero is denoted by $\varnothing$. We denote the composition $\left(\alpha_{\ell}, \ldots, \alpha_{1}\right)$ by $\alpha^{r}$. Also, denote the transpose of a partition $\lambda$ by $\lambda^{t}$. The reader is warned that on occasion we will suppress commas and parentheses when writing our compositions.

The composition diagram of $\alpha=\left(\alpha_{1}, \ldots, \alpha_{\ell}\right)$ is the left-justified array of boxes with $\alpha_{i}$ boxes in row $i$ from the bottom. If $\alpha$ is a partition, then the composition diagram of $\alpha$ coincides with the Young diagram of $\alpha$ in French notation. See Figure 1 for the composition diagram of $(2,1,3)$.

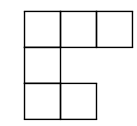

Figure 1. The composition diagram of $(2,1,3)$. 
2.3. Young tableaux. A Young tableau $T$ (henceforth YT) of skew shape $\lambda / \mu$ is a filling of the boxes of $\lambda / \mu$ with positive integers so that entries along the rows increase weakly read from left to right and entries along the columns increase strictly read from bottom to top. If the entries in $T$ are all distinct and belong to $[|\lambda / \mu|]$, then we call $T$ a standard Young tableau (henceforth SYT.) We denote the set of YTs of shape $\lambda / \mu$ by $\mathrm{YT}(\lambda / \mu)$.

If $T$ is a YT with maximal entry $m$, then the content of $T$, denoted by cont $(T)$, is the weak composition $\left(\alpha_{1}, \ldots, \alpha_{m}\right)$ where $\alpha_{i}$ for $1 \leqslant i \leqslant m$ counts the instances of $i$ in $T$. The standardization of $T$, denoted by $\operatorname{stan}(T)$, is obtained by replacing the $\alpha_{i}$ entries in $T$ equal to $i$ by the integers $1+\sum_{j=1}^{i-1} \alpha_{j}$ through $\sum_{j=1}^{i} \alpha_{j}$ from left to right.
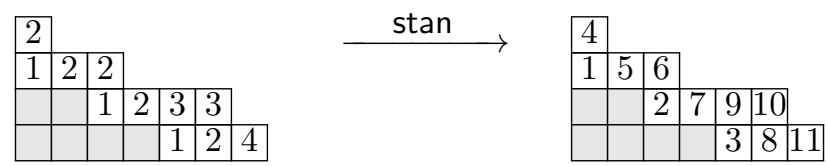

Figure 2. A Young tableau and its standardization.

2.4. Composition tableaux. To define composition tableaux, we need an analogue of Young's lattice. The Young composition poset $\mathcal{L}_{c}$ is the poset on compositions where the partial order $<_{c}$ is obtained by taking the transitive closure of the cover relation $\lessdot_{c}$ defined next. Let $\beta=\left(\beta_{1}, \ldots, \beta_{m}\right)$. Then $\beta \lessdot_{c} \alpha$ if exactly one of the following conditions holds.

- $\alpha=\left(\beta_{1}, \ldots, \beta_{m}, 1\right)$.

- $\alpha=\left(\beta_{1}, \ldots, \beta_{k}+1, \ldots, \beta_{m}\right)$ for some $k$ where $\beta_{k} \neq \beta_{i}$ for all $i>k$.

The reader may check that, for instance, the compositions covering $(2,1,3,2)$ in $\mathcal{L}_{c}$ are $(2,1,3,2,1),(2,2,3,2),(2,1,3,3)$ and $(2,1,4,2)$.

REMARK 2.1. The definition of $\lessdot_{c}$ implies that $\beta<_{c} \alpha$ if and only if for every $\beta_{i} \geqslant \beta_{j}$ where $i>j$ we have $\alpha_{i} \geqslant \alpha_{j}$.

If $\beta<_{c} \alpha$ and $\beta$ is drawn in the bottom left corner of $\alpha$, then the skew composition shape $\alpha / / \beta$ is defined to be the array of boxes that belong to $\alpha$ but not to $\beta$. We refer to $\alpha$ and $\beta$ as the outer shape and inner shape respectively. If the inner shape is $\varnothing$, then instead of writing $\alpha / / \varnothing$ we simply write $\alpha$. The size of $\alpha / / \beta$, denoted by $|\alpha / / \beta|$, is $|\alpha|-|\beta|$.

A composition tableau (abbreviated to CT) $\tau$ of shape $\alpha / / \beta$ is a filling $\tau: \alpha / / \beta \longrightarrow$ $\mathbb{Z}_{+}$that satisfies the following conditions.

(1) The entries in each row increase weakly from left to right.

(2) The entries in the leftmost column increase strictly from bottom to top.

(3) For any configuration in $\tau$ of the type in Figure 3, if $a \leqslant c$ then $b<c$.

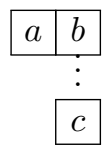

Figure 3. A triple configuration.

A composition tableau is standard if $\tau$ is a bijection between $\alpha / / \beta$ and $[|\alpha / / \beta|]$. We denote the set of CTs (respectively SCTs) of shape $\alpha / / \beta$ by $\mathrm{CT}(\alpha / / \beta)$ (respectively $\operatorname{SCT}(\alpha / / \beta))$. Figure 4 depicts a tableau in $\operatorname{CT}((3,6,1,7) / /(2,4))$, where the shaded boxes belong to the inner shape. 


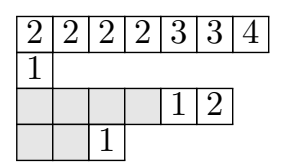

Figure 4. A CT of shape $(3,6,1,7) / /(2,4)$.

2.5. MASON's $\rho$ MAP. Next we discuss a crucial map that bridges the combinatorics of $\mathrm{CTs}$ and YTs. Let $\mathrm{CT}(-/ / \beta)$ denote the set of all $\mathrm{CTs}$ with inner shape $\beta$ and $\mathrm{YT}(-/ \widetilde{\beta})$ denote the set of all YTs with inner shape $\operatorname{sort}(\beta)$. Then the map $\rho_{\beta}: \mathrm{CT}(-\| \beta) \rightarrow$ $\mathrm{YT}(-/ \operatorname{sort}(\beta))$, which generalizes the map for semistandard skyline fillings [24] and is introduced in [22, Chapter 4], is defined as follows. Given $\tau \in \mathrm{CT}(-/ / \beta)$, obtain $\rho_{\beta}(\tau)$ by writing the entries in each column in increasing order from bottom to top and bottom-justifying these new columns on the inner shape $\operatorname{sort}(\beta)$, which might be empty.

The inverse map $\rho_{\beta}^{-1}: \mathrm{YT}(-/ \operatorname{sort}(\beta)) \rightarrow \mathrm{CT}(-/ / \beta)$ is also straightforward to define. Given $T \in \mathrm{YT}(-/ \operatorname{sort}(\beta))$,

(1) take the set of $i$ entries in the leftmost column of $T$ and write them in increasing order in rows $\ell(\beta)+1,2, \ldots, \ell(\beta)+i$ above the inner shape $\beta$ in the first column to form the leftmost column of $\tau$,

(2) take the set of entries in column 2 in increasing order and place them in the row with the largest index so that either

- the box to the immediate left of the number being placed is filled and the row entries weakly increase when read from left to right, or

- the box to the immediate left of the number being placed belongs to the inner shape,

(3) repeat the previous step with the set of entries in column $k$ for $k=3, \ldots, m$ where $m$ is the largest part of $\operatorname{sort}(\beta)$.

In the case $\beta=\varnothing$, the map $\rho_{\beta}$ is Mason's shift map [24] (or $\rho$ map), and we set $\rho:=\rho_{\varnothing}$. One may verify that the YT on the left in Figure 2 maps to the $\mathrm{CT}$ in Figure 4 under $\rho_{(2,4)}^{-1}$

2.6. REAding words, JDT-EQUivalenCE AND RECTIFICATIOn. Given a word $w=$ $w_{1} \cdots w_{n}$, the Robinson-Schensted correspondence (via row insertion or column insertion) associates an ordered pair $(\mathrm{P}(w), \mathrm{Q}(w))$ of YTs of the same shape. We call $\mathbf{P}(w)$ and $\mathbf{Q}(w)$ the insertion tableau and recording tableau respectively. We call two words $w_{1}$ and $w_{2}$ Knuth-equivalent if $\mathrm{P}\left(w_{1}\right)=\mathrm{P}\left(w_{2}\right)$. Intimately related to the notion of Knuth-equivalence is the notion of jdt-equivalence. For this we need to introduce reading words.

Definition 2.2 (Reading word). The reading word of $T \in \mathrm{YT}(\lambda / \mu)$ (respectively $\tau \in \mathrm{CT}(\alpha / / \beta)$ ), denoted by $\mathrm{rw}(T)$ (respectively $\mathrm{rw}(\tau)$ ), is obtained by reading the entries of $T$ (respectively $\tau$ ) in every column in decreasing order, starting from the leftmost column and going to the right.

We declare YTs $T_{1}$ and $T_{2}$ to be jdt-equivalent if their reading words are Knuthequivalent, that is, $\mathrm{P}\left(\mathrm{rw}\left(T_{1}\right)\right)=\mathrm{P}\left(\mathrm{rw}\left(T_{2}\right)\right)$. In view of Mason's map $\rho_{\beta}$, all combinatorial notions discussed in the context of Young tableaux are inherited by composition tableaux. We focus on the notion of rectification given its importance in our context.

Definition 2.3 (Rectification). Given $T \in \mathrm{YT}(\lambda / \mu)$, its rectification $\operatorname{rect}(T)$ is the unique $Y T$ of straight shape that is jdt-equivalent to $T$. This given, we can now define rectification for $C T s$. Given $\tau \in \mathrm{CT}(\alpha / / \beta)$, its rectification $\operatorname{rect}(\tau)$, is defined to be $\rho^{-1}\left(\operatorname{rect}\left(\rho_{\beta}(\tau)\right)\right)$. 
We make note of one important consequence: Let $\mu$ be a partition, and $\beta^{(1)}, \beta^{(2)}$ be compositions such that $\operatorname{sort}\left(\beta^{(i)}\right)=\mu$ for $i=1,2$. Given $T \in \mathrm{YT}(\lambda / \mu)$, let $\tau_{i}=$ $\rho_{\beta^{(i)}}^{-1}(T)$ for $i=1,2$. As $\operatorname{rw}\left(\tau_{1}\right)=\operatorname{rw}\left(\tau_{2}\right)$, we infer that $\operatorname{rect}\left(\tau_{1}\right)=\operatorname{rect}\left(\tau_{2}\right)$.

2.7. Classical LR Coefficients. Recall that the classical LR rule provides a combinatorial way to compute the structure coefficients $c_{\nu \mu}^{\lambda}$ in

$$
s_{\nu} s_{\mu}=\sum_{\lambda \vdash|\mu|+|\nu|} c_{\nu \mu}^{\lambda} s_{\lambda}
$$

The LR rule was stated by Littlewood-Richardson [19] in 1934, and the first proofs were obtained by Thomas [36] and Schützenberger [31] four decades later.

Definition 2.4. A tableau $T \in \mathrm{YT}(\lambda / \mu)$ is a Littlewood-Richardson tableau if $\operatorname{cont}(T)$ is a partition and $\operatorname{rw}(T)$ is reverse lattice. The set of LR tableaux of shape $\lambda / \mu$ and content $\nu$ is denoted by $\operatorname{LRT}(\lambda, \mu, \nu)$.

It transpires that the $\operatorname{LR}$ coefficient $c_{\nu \mu}^{\lambda}$ equals $|\operatorname{LRT}(\lambda, \mu, \nu)|$. Figure 5 depicts the LR tableaux that contribute to $c_{431,644}^{76432}$. For various combinatorial interpretations of LR coefficients, the reader is referred to [10, 38]. For a Hopf-algebraic perspective, see [16], and for a beautiful unifying polytopal perspective, see [26].
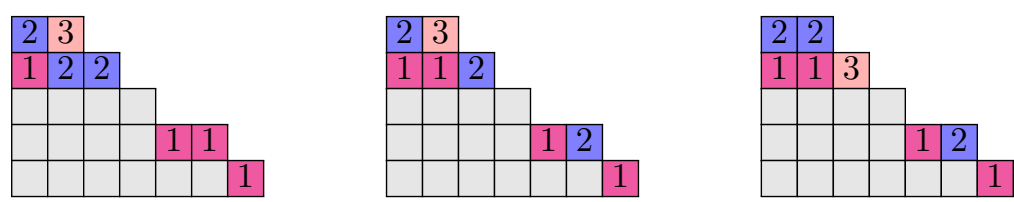

FiguRE 5. The three LR tableaux contributing to $c_{431,644}^{76432}$.

2.8. Noncommutative LR Coefficients. We refer the reader to $[12,13,22]$ for background on noncommutative symmetric functions and quasisymmetric functions. To describe the noncommutative LR rule, we need noncommutative analogues of Schur functions or, equivalently, quasisymmetric analogues of skew Schur functions. Following [22, Proposition 5.2.6], we define the skew quasisymmetric Schur function indexed by $\alpha / / \beta$ to be

$$
\mathcal{S}_{\alpha / / \beta}:=\sum_{\tau \in \mathrm{CT}(\alpha / / \beta)} \mathbf{x}^{\operatorname{cont}(\tau)} .
$$

Here $\mathbf{x}^{\operatorname{cont}(\tau)}:=x_{1}^{\alpha_{1}} \cdots x_{m}^{\alpha_{m}}$ where $m$ is the largest entry in $\tau$ and $\left(\alpha_{1}, \ldots, \alpha_{m}\right)=$ $\operatorname{cont}(\tau)$. If $\beta=\varnothing$ in (5), instead of writing $\mathcal{S}_{\alpha / / \varnothing}$, we write $\mathcal{S}_{\alpha}$. Additionally, we set $\mathcal{S}_{\varnothing}=1$.

The noncommutative Schur functions are defined indirectly [22, Definition 5.6.1] as elements of the basis in NSym dual to the basis of quasisymmetric Schur functions in QSym. We now proceed to describe the LR rule for noncommutative Schur functions, equivalent to [22, Theorem 5.6.2].

Given a composition $\alpha=\left(\alpha_{1}, \ldots, \alpha_{k}\right)$, the canonical composition tableau $\tau_{\alpha}$ is constructed by filling the boxes in the $i$-th row of the composition diagram of $\alpha$ with consecutive positive integers from $1+\sum_{j=1}^{i-1} \alpha_{j}$ to $\sum_{j=1}^{i} \alpha_{j}$ from left to right, for $1 \leqslant i \leqslant k$. Figure 6 shows the canonical CT of shape $(4,2,3,1)$ on the right.

Theorem 2.5. Let $\alpha, \beta$ be compositions. Then

$$
\mathbf{s}_{\alpha} \mathbf{s}_{\beta}=\sum_{\gamma \vDash|\alpha|+|\beta|} C_{\alpha \beta}^{\gamma} \mathbf{s}_{\gamma},
$$




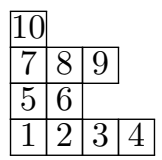

Figure 6 . The canonical CT of shape $(4,2,3,1)$.

where $C_{\alpha \beta}^{\gamma}$ is the number of SCTs of shape $\gamma / / \beta$ that rectify to $\tau_{\alpha}$.

For the proof of the theorem in its original context, see [5, Theorem 3.5]. As mentioned in the introduction, one motivation for this article is to develop a polytopal perspective on noncommutative LR coefficients. Toward this end, relating them explicitly with LR tableaux is a worthwhile endeavour as the latter's description allows for a straightforward translation to linear inequalities $[7,15,26]$.

2.9. Crystal reflection operators and LR tableaux. For in-depth exposition on crystal bases and their relevance in algebraic combinatorics and representation theory, we refer the reader to [8]. We proceed to describe crystal reflection operators.

Given a positive integer $i$, we define the crystal reflection operator $s_{i}$ acting on the set of Young tableaux as follows.

(1) Let $T \in \mathrm{YT}(\lambda / \mu)$, and let $w=w_{1} \ldots w_{n}:=\operatorname{rw}(T)$. Scan $w$ from left to right and pair each $i+1$ with the closest unpaired $i$ that follows.

(2) If no further pairing is possible, then change all unpaired $i$ 's to $i+1$ 's or vice versa depending on whether the number of $i$ 's is greater than the number of $i+1$ 's or not. Say the new word obtained via this procedure is $w^{\prime}$.

(3) Define $s_{i}(T)$ to be the unique YT of shape $\lambda / \mu$ such that $r w\left(s_{i}(T)\right)=w^{\prime}$.

Lascoux-Schützenberger [17] (see also [18, Section 3] and [28, Proposition 9]) proved that the operators $s_{i}$ give a well-defined action of the (infinite) symmetric group on $\mathrm{YT}(\lambda / \mu)$ by verifying the Coxeter-Moore relations. In particular, we obtain $\sigma(T)$ by computing $s_{i_{1}} \cdots s_{i_{k}}(T)$ for any reduced word $s_{i_{1}} \cdots s_{i_{k}}$ for $\sigma$.

For $\sigma \in \mathfrak{S}_{\ell(\nu)}$, define

$$
\operatorname{LRT}^{\sigma}(\lambda, \mu, \nu):=\{\sigma(T) \mid T \in \operatorname{LRT}(\lambda, \mu, \nu)\} .
$$

Since crystal reflection operators define an $\mathfrak{S}_{|\ell(\nu)|}$-action, we have $\left|\operatorname{LRT}^{\sigma}(\lambda, \mu, \nu)\right|$ equals $c_{\nu \mu}^{\lambda}$, for all permutations $\sigma \in \mathfrak{S}_{\ell(\nu)}$.

Figure 7 shows all tableaux in $\operatorname{LRT}^{\sigma}(\lambda, \mu, \nu)$ where $\lambda=(7,6,4,3,2), \mu=(6,4,4)$, $\nu=(4,3,1)$ and $\sigma=s_{1} s_{2}$. The tableaux in $\operatorname{LRT}(\lambda, \mu, \nu)$ are shown in Figure 5 . Note that all tableaux in Figure 7 have content $(1,4,3)=\sigma \cdot(4,3,1)$.
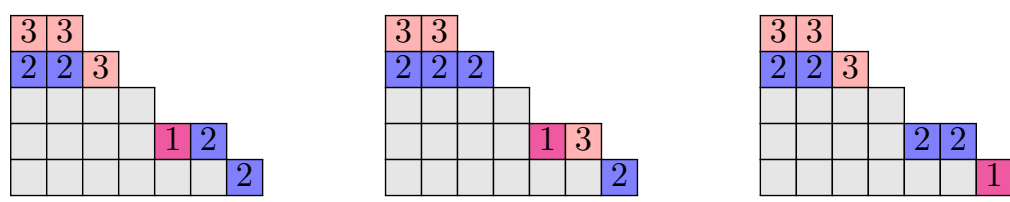

FiguRE 7. Crystal reflection corresponding to $s_{1} s_{2}$ on tableaux in Figure 5.

The crucial insight behind our work is that even though $\operatorname{LRT}^{\sigma}(\lambda, \mu, \nu)$ sheds no further light on classical LR coefficients, it "knows" about noncommutative LR coefficients. To motivate our upcoming results and to establish a connection with Theorem 2.5, we invite the reader to check that for any tableau $T$ in Figure 7, we have that $\rho^{-1}(\operatorname{rect}(T))$ is the distinguished $\mathrm{CT}$ in Figure 8 with the defining property that it is the unique $\mathrm{CT}$ with shape and content both equaling $(1,4,3)$. Equivalently, we could say that $\rho^{-1}(\operatorname{rect}(\operatorname{stan}(T)))$ is the canonical CT of shape $(1,4,3)$. 
Figure 8. The rectification of all tableaux in Figure 7.

In Section 5, we will establish this property in general by exploiting the interplay between frank words and jeu-de-taquin. This will allow us to establish the veracity of our main theorem.

\section{Main Result AND Related Discussion}

Our main result provides a combinatorial description for $C_{\alpha \beta}^{\gamma}$ using crystal reflection operators. We state it next and give a proof assuming results from Sections 4 and 5 .

THEOREM 3.1. Let $\alpha, \beta$ and $\gamma$ be compositions such that $|\beta|+|\alpha|=|\gamma|$. Let $(\lambda, \mu, \nu)$ be the triple $(\operatorname{sort}(\gamma), \operatorname{sort}(\beta), \operatorname{sort}(\alpha))$. If $\sigma \in \mathfrak{S}_{\ell(\nu)}$ is a permutation such that $\alpha=\sigma \cdot \nu$, then

$$
C_{\alpha \beta}^{\gamma}=\left|\left\{T \in \operatorname{LRT}^{\sigma}(\lambda, \mu, \nu) \mid \operatorname{shape}\left(\rho_{\beta}^{-1}(T)\right)=\gamma / / \beta\right\}\right| .
$$

Proof. Let $(\lambda, \mu, \nu)$ be as in the statement of the theorem. Recall from (3) in the introduction that

$$
c_{\nu \mu}^{\lambda}=\sum_{\operatorname{sort}(\delta)=\lambda} C_{\alpha \beta}^{\delta},
$$

where we work with the convention that $C_{\alpha \beta}^{\delta}=0$ if $\delta$ does not lie above $\beta$ in $\mathcal{L}_{c}$. Given $\gamma$ such that sort $(\gamma)=\lambda$, define

$$
X_{\gamma}:=\left\{T \in \operatorname{LRT}^{\sigma}(\lambda, \mu, \nu) \mid \operatorname{shape}\left(\rho_{\beta}^{-1}(\operatorname{stan}(T))\right)=\gamma\right\} .
$$

By Proposition 5.3, we have that $\left|X_{\gamma}\right| \leqslant C_{\alpha \beta}^{\gamma}$. On the other hand, from (7) and the fact that shape $\left(\rho_{\beta}^{-1}(\operatorname{stan}(T))\right)=\operatorname{shape}\left(\rho_{\beta}^{-1}(T)\right)$ for all YTs $T$ with inner shape $\mu$, we infer that

$$
\left|\operatorname{LRT}^{\sigma}(\lambda, \mu, \nu)\right|=\sum_{\operatorname{sort}(\delta)=\lambda}\left|X_{\delta}\right| .
$$

Thus, we must have $\left|X_{\gamma}\right|=C_{\alpha \beta}^{\gamma}$.

3.1. Comparison With KNOWn Results. It is natural to inquire whether our Theorem 3.1 affords further insight into computing noncommutative LR coefficients when compared to the existing description in [5]. To this end, note that [22, Theorem 5.6.2] offers little algorithmic insight in terms of judiciously picking SCTs of shape $\gamma / / \beta$ that rectify to $\tau_{\alpha}$, other than an exhaustive search. Additionally, given the involved bumping algorithm for jeu-de-taquin on composition tableaux [34], the efficient approach to rectification is still Mason's insertion algorithm.

In contrast, Theorem 3.1 emphasizes the curious aspect that computing noncommutative LR coefficients is best done by passing through the commutative realm. Indeed, although computing classical LR coefficients is known to be \#P-hard, we can appeal to one of many descriptions that they possess to compute them in a deterministic manner; see $[7,15,20,37]$. Once the tableaux in $\operatorname{LRT}(\lambda, \mu, \nu)$ are computed, it remains to apply an appropriate sequence of crystal reflections (determined by $\alpha$ ) followed by an appropriate $\rho_{\beta}$ to determine all noncommutative LR coefficients $C_{\alpha \beta}^{\gamma}$.

On a related noted, Bessenrodt-Tewari-van Willigenburg [6], in classifying symmetric skew quasisymmetric Schur functions, established Yamanouchi-type rules called left and right LR rules therein - for the $C_{\alpha \beta}^{\gamma}$ in the special cases where $\alpha$ is either a partition or a reverse partition, although the proof therein was not uniform. 
It transpires that these rules are in fact two extremes of our Theorem 3.1. Indeed by picking $\sigma$ to either be the identity or the longest word in $\mathfrak{S}_{\ell(\nu)}$, we obtain the rules of $[6]$; see discussion at the end of Section 5 for details.

3.2. Illustrating OUR MAIN RESUlt. We discuss an example next to illustrate Theorem 3.1. Let $\lambda=8642, \mu=4321$ and $\nu=532$. Figure 9 shows tableaux in $\operatorname{LRT}(\lambda, \mu, \nu)$, implying in particular that $c_{532,4321}^{8642}=4$.
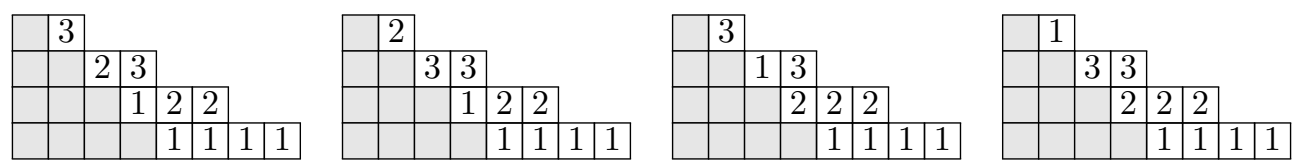

Figure 9. The tableaux in $\operatorname{LRT}(8642,4321,532)$.

EXAMPLE 3.2. Suppose we pick $\sigma \in \mathfrak{S}_{3}$ to be the identity permutation and $\beta=\mu$. Thus we do not need to apply crystal reflections. By applying $\rho_{4321}^{-1}$ to the LR tableaux in Figure 9 we obtain the CTs in Figure 10. By grouping these CTs according to outer shape, we infer that

$$
C_{532,4321}^{6842}=C_{532,4321}^{6824}=C_{532,4321}^{8462}=C_{532,4321}^{8624}=1 .
$$

In particular we have $C_{532,4321}^{\gamma}=0$ for all rearrangements of $\lambda$ that are not in $\{6842,6824,8462,8624\}$.
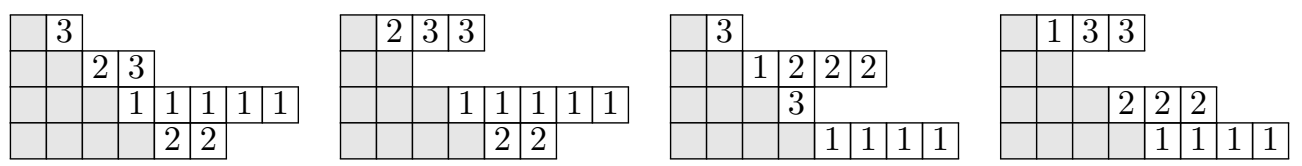

Figure 10. CTs obtained by applying $\rho_{4321}^{-1}$ to tableaux in Figure 9.

EXAMPLE 3.3. Sticking with the same $\sigma$ as before, we now pick $\beta=3412$. The CTs resulting from applying $\rho_{3412}^{-1}$ are shown in Figure 11. Grouping them according to outer shape reveals a different decomposition of $c_{532,4321}^{8642}$. More precisely, we obtain

$$
C_{532,3412}^{6824}=3, \text { and } C_{532,3412}^{4826}=1 .
$$
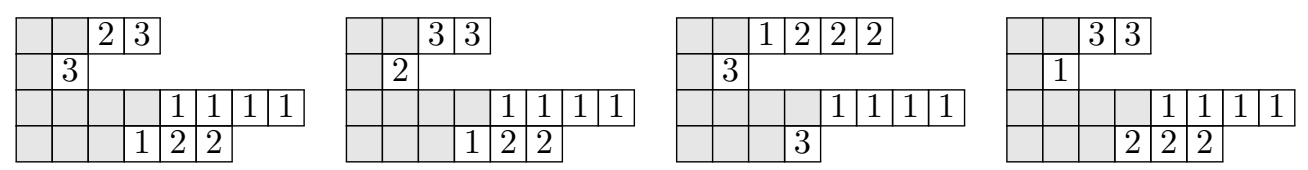

Figure 11. CTs obtained by applying $\rho_{3412}^{-1}$ to tableaux in Figure 9.

EXAMPle 3.4. Suppose we now pick $\sigma=s_{1} s_{2}$. Then $\alpha=\sigma \cdot \nu=(2,5,3)$ and the four tableaux in $\operatorname{LRT}^{\sigma}(\lambda, \mu, \nu)$ are depicted in Figure 12. We pick $\beta=1243$. Applying $\rho_{1243}^{-1}$ to these tableaux gives us the CTs in Figure 13. This time we obtain

$$
C_{253,1243}^{2468}=2 \text {, and } C_{253,1243}^{2486}=2 .
$$



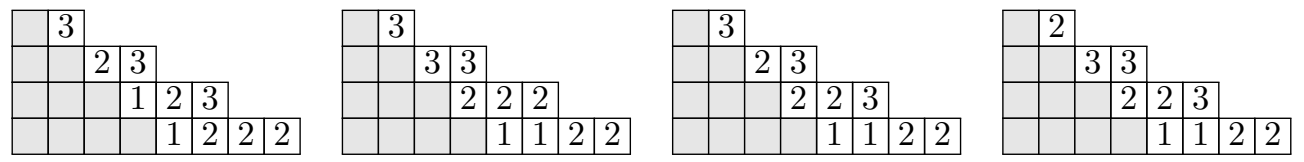

Figure 12. The action of $s_{1} s_{2}$ on tableaux in Figure 9.
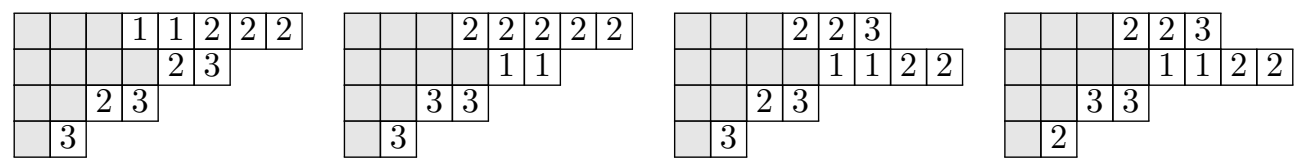

Figure 13. CTs obtained by applying $\rho_{1243}^{-1}$ to tableaux in Figure 12.

\section{Frank WORdS AND LR tableaux}

In order to prove Theorem 3.1, we need to understand the rectification of tableaux in $\operatorname{LRT}^{\sigma}(\lambda, \mu, \nu)$ followed by an application of the generalized $\rho$ map. To this end, it helps to explore the relation between growth words of these tableaux and frank words.

\subsection{GROWTH WORDS AND FRANK WORDS.}

DEFInITION 4.1 (Growth word). Given an $S Y T T$, its growth word $\operatorname{gw}(T)$ is obtained by reading entries from largest to smallest and recording the columns they belong to. We extend the definition of growth word to all YTs by setting $\operatorname{gw}(T):=\operatorname{gw}(\operatorname{stan}(T))$.

The reader may verify that for both tableaux in Figure 2, the growth word is 76564321531 .

Frank words were introduced by Lascoux-Schützenberger [18] in their investigation of key polynomials. Subsequently, Reiner and Shimozono [27] studied the combinatorics of frank words in depth in the context of a flagged LR rule, and we follow their exposition as far as notions in this section are concerned.

We call $w=w_{1} \cdots w_{n} \in \mathbb{Z}_{+}^{*}$ a column word if $w_{1}>\cdots>w_{n}$. Given a nonempty word $w \in \mathbb{Z}_{+}^{*}$, consider its factorization $w^{(1)} w^{(2)} \cdots w^{(m)}$ where each $w^{(i)}$ is a maximal column word (necessarily nonempty). We call $w$ an m-column word. The column form of $w$ is the composition colform $(w):=\left(\left|w^{(1)}\right|, \ldots,\left|w^{(m)}\right|\right)$.

Definition 4.2 (Frank word). We say that $w$ is frank if $\mathrm{P}(w)$ is of shape $\lambda^{t}$ where $\lambda=\operatorname{sort}(\operatorname{colform}(w))$. Given a composition $\alpha$, we define $\operatorname{Frank}(\alpha)$ to be the set of frank words $w$ satisfying colform $(w)=\alpha$.

A remarkable feature of frank words is that one knows the shape of the P-tableau without performing jeu-de-taquin. As an example of a frank word, consider $w=$ 432326531 . It is a 3 -column word with colform $(w)=(3,2,4)$. For clarity, we put frames around maximal column words. Figure 14 depicts $\mathrm{P}(w)$. Note that the shape underlying it is $(4,3,2)^{t}$. Therefore, $w \in \operatorname{Frank}((3,2,4))$.

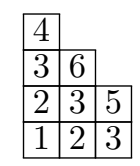

FIGURE 14. The insertion tableau corresponding to $w=432326531$. 
4.2. OUtLINE OF PROOF. Given the technical nature of our argument, we give a brief overview of our strategy toward establishing Theorem 3.1.

(1) Given a skew shape $\lambda / \mu$ and a composition $\alpha \vDash|\lambda|-|\mu|$, we consider the distinguished subset of $\operatorname{Frank}(\alpha)$ comprising $\lambda / \mu$-compatible frank words.

(2) The main result of this section, Proposition 4.6, identifies these words as precisely the growth words of tableaux in $\operatorname{LRT}^{\sigma}(\lambda, \mu, \nu)$ where $\nu$ satisfies $\operatorname{sort}(\alpha)=\nu$. To arrive at this result, we study a symmetric group action on frank words and establish that it really is the crystal reflection action in disguise. This is the content of Subsections 4.4 and 4.5.

(3) Lemma 5.1 then states that the rectification of a Young tableau may be described in terms of its growth word using Schützenberger's evacuation.

(4) Finally, Proposition 5.3 establishes that applying $\rho^{-1}$ to rect $(\operatorname{stan}(T))$ for $T$ an element in $\operatorname{LRT}^{\sigma}(\lambda, \mu, \nu)$ does indeed give the canonical tableau of shape $\sigma \cdot \nu$. From this, Theorem 3.1 follows.

\section{3. $\lambda / \mu$-COMPATIBLE FRANK WORDS AND LR TABLEAUX.}

Definition 4.3. Given partitions $\lambda, \mu$ such that $\mu \subseteq \lambda$ and a composition $\alpha$, we say that $w \in \operatorname{Frank}(\alpha)$ is $\lambda / \mu$-compatible if for every suffix $w^{\prime}$ of $w$, we have that $\operatorname{cont}\left(w^{\prime}\right)+\mu^{t}$ is a partition and that $\operatorname{cont}(w)+\mu^{t}=\lambda^{t}$. Set

$$
\operatorname{Frank}(\lambda, \mu, \alpha):=\{w \in \operatorname{Frank}(\alpha) \mid w \text { is } \lambda / \mu \text {-compatible }\} .
$$

Definition 4.3 may be interpreted as saying that $w \in \operatorname{Frank}(\alpha)$ is $\lambda / \mu$-compatible if it is the growth word of a Young tableau of shape $\lambda / \mu$. Observe that we must have $\alpha \vDash|\lambda|-|\mu|$.

As an example, note that $w=62176432 \in \operatorname{Frank}(\alpha)$ is $\lambda / \mu$-compatible for $\lambda=76422, \mu=5521$, and $\alpha=35$. The reader can easily verify that $w$ is the growth word of the tableau in Figure 15. Note further that this tableau is an LR tableau.

There is an intimate link between LR tableaux and compatible words. To understand this link we go via an intermediate tableau object.

Given $w \in \operatorname{Frank}(\lambda, \mu, \alpha)$, let $w^{(1)} \cdots w^{(m)}$ be its maximal column word factorization where $m:=\ell(\alpha)$. Construct a Young tableau $\phi(w)$ of shape $\lambda / \mu$ and content $\alpha^{r}$ as follows: Let $\lambda^{(0)}:=\lambda$ and inductively define $\lambda^{(i)}$ for $1 \leqslant i \leqslant m$ to be such that $\lambda^{(i-1)} / \lambda^{(i)}$ is a horizontal strip with boxes in columns given by letters appearing in $w^{(i)}$. Subsequently, fill the boxes of the horizontal strips $\lambda^{(i-1)} / \lambda^{(i)}$ with $m+1-i$ to obtain $\phi(w)$. Note that $\lambda^{(m)}$ is $\mu$ and that $\phi(w)$ does indeed belong to $\operatorname{YT}(\lambda / \mu)$. Figure 15 gives an example.

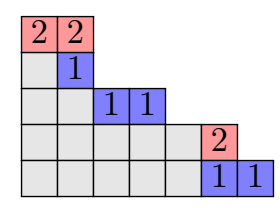

FigURE 15. The tableau $\phi(w)$ corresponding to 62176432 .

Define

$$
\operatorname{FrankTab}(\lambda, \mu, \alpha):=\{\phi(w) \mid w \in \operatorname{Frank}(\lambda, \mu, \alpha)\} .
$$

Our next lemma establishes that $\operatorname{LRT}(\lambda, \mu, \nu)=\operatorname{FrankTab}\left(\lambda, \mu, \nu^{r}\right)$.

Lemma 4.4. Let $\lambda, \mu$ and $\nu$ be partitions such that $\mu \subseteq \lambda$ and $|\nu|=|\lambda / \mu|$. We have

$$
\operatorname{LRT}(\lambda, \mu, \nu)=\operatorname{FrankTab}\left(\lambda, \mu, \nu^{r}\right) .
$$


Proof. Let $w \in \operatorname{Frank}\left(\lambda, \mu, \nu^{r}\right)$. As colform $(w)=\nu^{r}$ and $w$ is frank, we know that $w$ is the reading word of a tableau of skew shape rotate $\left(\nu^{t}\right)$, which is obtained from the shape $\nu$ by a $180^{\circ}$ rotation. It follows that $\phi(w) \in \operatorname{LRT}(\lambda, \mu, \nu)$. This establishes $\operatorname{FrankTab}\left(\lambda, \mu, \nu^{r}\right) \subseteq \operatorname{LRT}(\lambda, \mu, \nu)$.

The opposite inclusion follows since the reading word of an LR tableau is reverselattice. In particular, the growth word of any $T \in \operatorname{LRT}(\lambda, \mu, \nu)$ is the reading word of a YT of skew shape rotate $\left(\nu^{t}\right)$.

Figure 16 depicts growth words of LR tableaux in Figure 5 as reading words of YTs of skew shape rotate $\left(\nu^{t}\right)$. Here $\lambda=76432, \mu=644$ and $\nu=431$.
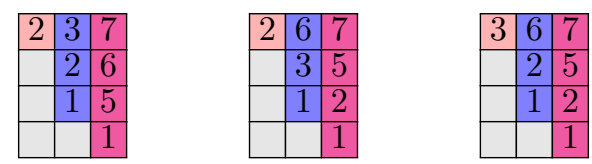

FiguRE 16. Growth words of LR tableaux are frank words.

Now that we have established elements of $\operatorname{FrankTab}\left(\lambda, \mu, \nu^{r}\right)$ as LR tableaux in the usual sense, it remains to understand the case where $\nu^{r}$ is replaced by an arbitrary composition.

4.4. A SYMMETRIC GROUP ACTION ON FRANK WORDS. We proceed to describe a symmetric group action on frank words that we subsequently connect to the symmetric group action on Young tableaux via crystal reflections described earlier. This new action is best understood by focusing on 2-column frank words, very much in the manner that crystal reflections are understood by acting on consecutive letters.

Let $A$ denote the set of 2-column frank words. Consider $w \in A$ and let colform $(w)=$ $\left(\beta_{1}, \beta_{2}\right)$. Thus, $w$ may be identified as the reading word of a tableau $T$ of shape $\left(\beta_{1}, \beta_{2}\right)^{t}$ if $\beta_{1} \geqslant \beta_{2}$ or of a tableau $T$ of shape $\left(\beta_{2}, \beta_{2}\right)^{t} /\left(\beta_{2}-\beta_{1}\right)^{t}$ if $\beta_{1}<\beta_{2}$; see [27, Appendix 2].

If the former holds, define $\iota(w)$ to be the reading word of the unique tableau $T^{\prime}$ of shape $\left(\beta_{1}, \beta_{1}\right)^{t} /\left(\beta_{1}-\beta_{2}\right)^{t}$ that is jdt-equivalent to $T$ (obtained by performing jeu-detaquin slides within the rectangle $\left.\left(\beta_{1}, \beta_{1}\right)^{t}\right)$. If the latter holds, define $\iota(w)$ to be the reading word of the unique tableau $T^{\prime}$ of shape $\left(\beta_{2}, \beta_{1}\right)^{t}$ that is jdt-equivalent to $T$.

Clearly, $\iota$ is an involution on A. Equally importantly, $w$ and $\iota(w)$ are Knuthequivalent. For instance, jdt-equivalence of tableaux in Figure 17 implies that

$$
\iota(76421632)=62166432 .
$$

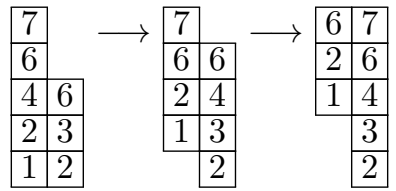

Figure 17. Establishing $\iota(76421632)=621 \quad 76432$ by jeu-de-taquin.

We employ the involution $\iota$ to construct the desired symmetric group action. Let $\lambda$ be a partition and let $m:=\ell(\lambda)$. Define

$$
\mathcal{F}_{\lambda}:=\underset{\operatorname{sort}(\beta)=\lambda}{\coprod} \operatorname{Frank}(\beta) .
$$


Following [18], define an action of $\mathfrak{S}_{m}$ on $\mathcal{F}_{\lambda}$ by describing the action of the generator $s_{i}$ for $1 \leqslant i \leqslant m-1$ as follows: Let $w^{(1)} \cdots w^{(m)}$ be the maximal column word factorization of $w \in \mathcal{F}_{\lambda}$. Let $v^{(i)} v^{(i+1)}:=\iota\left(w^{(i)} w^{(i+1)}\right)$ and define $v=$ $w^{(1)} \cdots w^{(i-1)} v^{(i)} v^{(i+1)} w^{(i+2)} \cdots w^{(m)}$. Observe that sort (colform $\left.(v)\right)=\lambda$. As $v$ and $w$ are Knuth-equivalent, we infer that $v \in \mathcal{F}_{\lambda}$. We define $v$ to be $s_{i}(w)$. This given, we may define $\sigma(w)$ for any $\sigma \in \mathfrak{S}_{m}$ by picking a reduced word for $\sigma$.

Figure 18 depicts the $\mathfrak{S}_{3}$-orbit of the frank word $4321632 \quad 53 \in \mathcal{F}_{432}$. We have chosen to represent the action on words via their tableau representatives. In particular, each column when read from top to bottom gives a maximal column word in the maximal column word factorization. Each $s_{i}$ performs a "local" jeu-de-taquin on two columns.

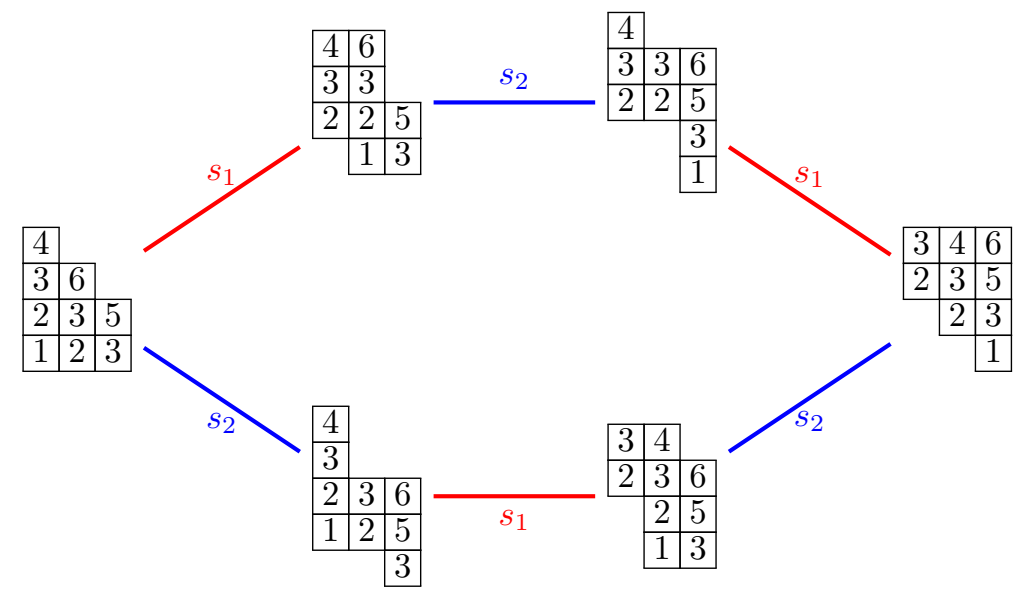

Figure 18. The $\mathfrak{S}_{3}$-orbit of 432163253.

4.5. Relating the two symmetric group actions. Our next lemma connects the action of crystal reflection operators on $\operatorname{Frank} \operatorname{Tab}(\lambda, \mu, \alpha)$ to the symmetric group action on $\lambda / \mu$-compatible frank words in $\operatorname{Frank}(\lambda, \mu, \alpha)$.

Lemma 4.5. Consider $w \in \operatorname{Frank}(\lambda, \mu, \alpha)$ and $i$ satisfying $1 \leqslant i \leqslant \ell(\alpha)-1$. We have that $s_{i}(\phi(w))=\phi\left(s_{\ell(\alpha)-i}(w)\right)$.

Proof. Without loss of generality, we may assume that $\alpha$ has two parts. Suppose $\alpha=(p, q) \vDash n$. Assume $w=w^{(1)} w^{(2)}$ where $\left|w^{(1)}\right|=p$ and $\left|w^{(2)}\right|=q$. We want to show that $s_{1}(\phi(w))=\phi(\iota(w))$.

Assume $p<q$. Let $w^{(1)}=a_{1} \ldots a_{p}$ and $w^{(2)}=b_{1} \ldots b_{q}$. We have $a_{1}>\cdots>a_{p}$ and $b_{1}>\cdots>b_{q}$. As $w$ is frank, it is the reading word of a Young tableau of skew shape rotate $\left((q, p)^{t}\right)$. Thus, $a_{i} \leqslant b_{i}$ for $1 \leqslant i \leqslant p$.

Instead of computing $\iota(w)$ by rectifying the appropriate two-columned tableau, one may perform successive Schensted column insertions of the numbers $a_{p}$ down to $a_{1}$ starting from the single-columned tableau with column word $w^{(2)}$. See Figure 19 for an example. Compare with Figure 17 which established the same fact using jeu-de-taquin.

In each intermediate step of this column insertion procedure, the number $a_{i}$ being inserted into the current tableau bumps a distinct element from $\left\{b_{1}, \ldots, b_{q}\right\}$. Furthermore this bumped entry is guaranteed to be strictly greater than the entries in the second column in the current tableau. Therefore, the insertion tableau is completely determined by the entries that get bumped. More precisely, for $i$ from $p$ down to 1 , define 
Figure 19. Establishing that $\iota(62176432)=76421632$ by column-inserting 1,2 , and 6 into the single-columned tableau with reading word 76432 . Blue boxes show the entries being inserted whereas red boxes contain the entries bumped.

the integer $\mathrm{m}(i)$ recursively as follows. We define $\mathrm{m}(p)$ to be the largest integer $j$ such that $a_{p} \leqslant b_{j}$. Subsequently, for $i=p-1, \ldots, 1$, define $\mathrm{m}(i)$ to be the largest integer $j$ such that $j<\mathrm{m}(i+1)$ and $a_{i} \leqslant b_{j}$. Observe that in our Schensted column-insertion procedure, the entry $a_{i}$ bumps $b_{\mathrm{m}(i)}$. Therefore, the set of entries that get bumped is $\left\{b_{\mathrm{m}(i)} \mid 1 \leqslant i \leqslant p\right\}$. For the example in Figure 19, we have $\mathrm{m}(3)=5, \mathrm{~m}(2)=4$ and $\mathrm{m}(1)=2$. Therefore the set of entries that get bumped is $\left\{b_{5}, b_{4}, b_{2}\right\}=\{2,3,6\}$.

Consider $\operatorname{rw}(\phi(w))=u_{1} \cdots u_{n}$. The word $v:=v_{1} \cdots v_{n}$ obtained by recording the column to which each $u_{i}$ belongs gives us the weakly increasing arrangement of letters in $w$. Furthermore, for $1 \leqslant i \leqslant p$ (respectively $1 \leqslant i \leqslant q$ ) the letter in $v$ corresponding to the $i$ th 2 (respectively 1 ) from the left in $\operatorname{rw}(\phi(w))$ is equal to $a_{i}$ (respectively $b_{i}$ ). Recall that the crystal reflection operator $s_{1}$ acting on $\phi(w)$ begins by pairing each 2 in $\operatorname{rw}(\phi(w))$ to the closest unpaired 1 to its right. Equivalently, in our current context, a 2 corresponding to $a_{i}$ for some $1 \leqslant i \leqslant p$ gets paired with the 1 in $\operatorname{rw}(\phi(w))$ corresponding to $b_{\mathrm{m}(i)}$. We infer that the unpaired 1s in $\operatorname{rw}(\phi(w))$ correspond to those $b_{j}$ that are not bumped. These are precisely the $b_{j}$ that determine which $1 \mathrm{~s}$ in $\phi(w)$ turn into $2 \mathrm{~s}$ in computing $s_{1}(\phi(w))$. Thus we infer that $s_{1}(\phi(w))=\phi(\iota(w))$. This establishes the claim in the case $p<q$. The case $p \geqslant q$ is similar and left to the reader.

To illustrate the ideas in the preceding proof, Figure 20 depicts the action of $s_{1}$ on the tableau $\phi(w)$ from Figure 15, where $w=621$ 76432. From Figure 19, we see that the entries that do not get bumped are $\{4,7\}$. Also, note that $\operatorname{rw}(\phi(w))=22111211$ where the unpaired 1s are highlighted. In terms of the tableau $\phi(w)$, we see that the unpaired 1s belong to columns 4 and 7. The tableau on the right in Figure 20 is easily verified to be $\phi(\iota(w))$ as $\iota(w)=76421632$.
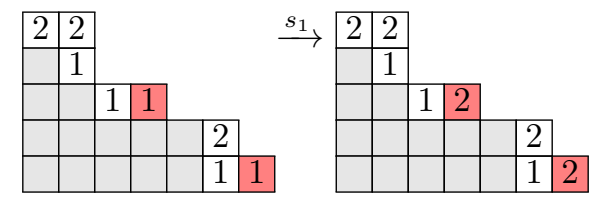

Figure 20. Crystal reflection on $\phi(w)$ and its relation to the $\iota$ involution.

We are ready to give a precise relation between $\operatorname{LRT}^{\sigma}(\lambda, \mu, \nu)$ and $\lambda / \mu$-compatible frank words with a certain column form that generalizes Lemma 4.4.

Proposition 4.6. Let $\lambda, \mu$ and $\nu$ be partitions such that $\mu \subseteq \lambda$ and $|\nu|=|\lambda / \mu|$. Let $w_{0}$ be the longest word in $\mathfrak{S}_{\ell(\nu)}$. For $\sigma \in \mathfrak{S}_{\ell(\nu)}$, we have

$$
\operatorname{LRT}^{\sigma}(\lambda, \mu, \nu)=\operatorname{FrankTab}\left(\lambda, \mu,\left(w_{0} \sigma w_{0}\right) \cdot \nu^{r}\right) .
$$

Proof. Lemma 4.4 implies

$$
\operatorname{LRT}(\lambda, \mu, \nu)=\operatorname{Frank} \operatorname{Tab}\left(\lambda, \mu, \nu^{r}\right) .
$$


Consider $T \in \operatorname{LRT}^{\sigma}(\lambda, \mu, \nu)$. We must have $T=\sigma\left(T^{\prime}\right)$ for a unique $T^{\prime} \in \operatorname{LRT}(\lambda, \mu, \nu)$, which in turn implies that $T=\sigma\left(\phi\left(w^{\prime}\right)\right)$ for a unique $w^{\prime} \in \operatorname{Frank}\left(\lambda, \mu, \nu^{r}\right)$. Lemma 4.5 implies that $T=\phi\left(w_{0} \sigma w_{0}\left(w^{\prime}\right)\right)$. As colform $\left(w_{0} \sigma w_{0}\left(w^{\prime}\right)\right)=\left(w_{0} \sigma w_{0}\right) \cdot \nu^{r}$, we conclude that

$$
\operatorname{LRT}^{\sigma}(\lambda, \mu, \nu) \subseteq \operatorname{FrankTab}\left(\lambda, \mu,\left(w_{0} \sigma w_{0}\right) \cdot \nu^{r}\right) .
$$

A simple cardinality count implies this inclusion must be an equality.

The upshot of Proposition 4.6 is that elements of $\operatorname{LRT}^{\sigma}(\lambda, \mu, \nu)$ are in bijection with $\lambda / \mu$-compatible frank words with column form $w_{0} \sigma w_{0} \cdot \nu^{r}$. As an example, consider $\operatorname{LRT}^{\sigma}(\lambda, \mu, \nu)$ from Figure 7 where $\lambda=(7,6,4,3,2), \mu=(6,4,4), \nu=(4,3,1)$ and $\sigma=s_{1} s_{2}$. Note that growth words of the tableaux therein are indeed frank words with column form $\left(w_{0} s_{1} s_{2} w_{0}\right) \cdot(1,3,4)=\left(s_{2} s_{1}\right) \cdot(1,3,4)=(3,4,1)$. Figure 21 shows these frank words as reading words of tableaux with column lengths 3,4 and 1 read from left to right. We encourage the reader to obtain these tableaux by performing jeu-de-taquin slides to those in Figure 16.

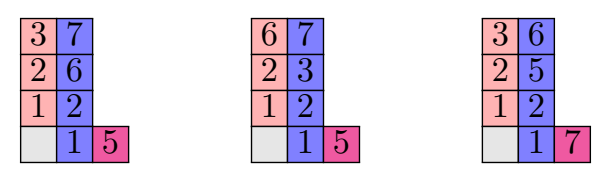

FiguRE 21. Growth words of tableaux in Figure 7 are frank words.

\section{Noncommutative LR COEfficients And FRAnK WORDS}

Now that we understand tableaux in $\operatorname{LRT}^{\sigma}(\lambda, \mu, \nu)$ as certain $\lambda / \mu$-compatible words with a prescribed column form, we are ready to establish the connection to noncommutative LR coefficients. Recall that our goal is to show that rect $(\operatorname{stan}(T))$ for $T \in \operatorname{LRT}^{\sigma}(\lambda, \mu, \nu)$ is equal to the canonical CT of shape $\sigma \cdot \nu$. We recast rect $(\operatorname{stan}(T))$ in terms of the growth word of $T$.

Lemma 5.1. For any Young tableau $T$, we have $\operatorname{rect}(\operatorname{stan}(T))=\operatorname{evac}(\mathrm{Q}(\operatorname{gw}(T)))^{t}$.

Proof. We sketch the proof and follow the exposition in [21]. Let $T^{\prime}=\operatorname{stan}(T)$ and suppose that $T$ has $n$ boxes. Consider the biword $\left[\begin{array}{l}u \\ v\end{array}\right]$ where $u:=u_{1} \cdots u_{n}$ is the longest word in $\mathfrak{S}_{n}$ and $v:=v_{1} \cdots v_{n}$ is obtained by recording the column in $T^{\prime}$ to which $u_{i}$ belongs. In other words, $v=\operatorname{gw}\left(T^{\prime}\right)=\operatorname{gw}(T)$. Consider biwords $\left[\begin{array}{c}u^{\prime} \\ v^{\prime}\end{array}\right]$ and $\left[\begin{array}{l}u^{\prime \prime} \\ v^{\prime \prime}\end{array}\right]$, where $u^{\prime}$ (respectively $v^{\prime \prime}$ ) in the weakly increasing rearrangement of $u$ (respectively $v$ ) and $v^{\prime}$ (respectively $u^{\prime \prime}$ ) is the rearrangement induced by the aforementioned sorting. Then we have that

$$
v^{\prime}=\operatorname{gw}\left(T^{\prime}\right)^{r} \text { and } u^{\prime \prime}=\operatorname{rw}\left(T^{\prime}\right) .
$$

Note that $\operatorname{rect}(\operatorname{stan}(T))=\operatorname{rect}\left(T^{\prime}\right)=\mathrm{P}\left(\operatorname{rw}\left(T^{\prime}\right)\right)=\mathrm{P}\left(u^{\prime \prime}\right)$. By [21, Proposition 5.3.9], this equals $\mathrm{Q}\left(v^{\prime}\right)$ and by [33, Corollary A1.2.11] which relates reversal to evacuation, the claim now follows.

For the leftmost tableau $T$ in Figure 22, its standardization rectifies to the tableau in the middle. We have $\operatorname{gw}(T)=3217621,5$, and $\mathrm{Q}(\mathrm{gw}(T))$ is shown on the right. We invite the reader to verify that evac $(\mathrm{Q}(\operatorname{gw}(T)))$ is indeed the tableau in the middle upon transposing.

We now appeal to a simple defining characterization of canonical composition tableaux that will be useful. Recall that the descent set of an SYT $T$ with $n$ boxes 


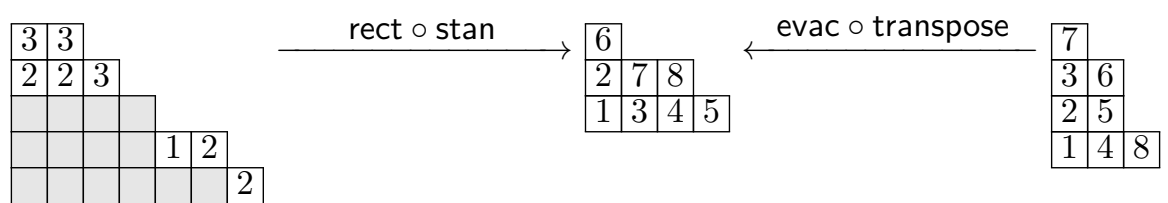

Figure 22. A demonstration of the claim $\operatorname{rect}(\operatorname{stan}(T))=\operatorname{evac}(\mathrm{Q}(\operatorname{gw}(T)))^{t}$.

is the set of integers $i \in[n-1]$ such that $i+1$ occupies a row strictly above that occupied by $i$. Via the folklore bijection between compositions of $n$ and subsets of $[n-1]$ that sends $\alpha=\left(\alpha_{1}, \ldots, \alpha_{\ell}\right)$ to $\operatorname{set}(\alpha):=\left\{\alpha_{1}, \alpha_{1}+\alpha_{2}, \ldots, \alpha_{1}+\cdots+\alpha_{\ell-1}\right\}$, one obtains the descent composition of $T$ from its descent set.

Lemma 5.2. Let $\alpha$ be a composition. There is a unique Young tableau $T$ of shape $\operatorname{sort}(\alpha)$ and descent composition $\alpha$. Furthermore, this tableau satisfies $\rho^{-1}(T)=\tau_{\alpha}$.

Proof. Observe that the first column of $T$ when read from bottom to top is forced to be $1,1+\alpha_{1}, 1+\alpha_{1}+\alpha_{2}, \ldots, 1+\sum_{j=1}^{\ell(\alpha)-1} \alpha_{j}$. The first claim follows from this observation, and our second claim follows from applying the map $\rho$ to $\tau_{\alpha}$. We omit the details.

We finally arrive at the key proposition that is utilized in the proof of our central result Theorem 3.1 .

Proposition 5.3. If $T \in \operatorname{LRT}^{\sigma}(\lambda, \mu, \nu)$, then $\operatorname{rect}(\operatorname{stan}(T))=\rho\left(\tau_{\alpha}\right)$ where $\alpha=\sigma \cdot \nu$.

Proof. Throughout, assume that $\sigma \cdot \nu=\alpha$ and set $n:=|\alpha|$. By Lemma 5.1 we have

$$
\operatorname{rect}(\operatorname{stan}(T))=\operatorname{evac}(\mathrm{Q}(\operatorname{gw}(T)))^{t} .
$$

Since $T \in \operatorname{LRT}^{\sigma}(\lambda, \mu, \nu)$, Proposition 4.6 implies

$$
\operatorname{gw}(T) \in \operatorname{Frank}\left(\lambda, \mu, \alpha^{r}\right) .
$$

Therefore, the shape underlying $\mathrm{Q}(\operatorname{gw}(T))$ is sort $(\alpha)^{t}=\nu^{t}$. Using the preceding fact along with (12), we infer that $\operatorname{rect}(\operatorname{stan}(T))$ has shape $\nu$.

Note that the descent set of $\operatorname{gw}(T)$ is $\{n-i \mid i \in[n-1] \backslash \operatorname{set}(\alpha)\}$, which therefore is also the descent set of $\mathrm{Q}(\operatorname{gw}(T))$. It follows that the descent set of evac $(\mathrm{Q}(\operatorname{gw}(T)))$ is $[n-1] \backslash \operatorname{set}(\alpha)$, which in turn implies that the descent set of $\operatorname{rect}(\operatorname{stan}(T))=$ $\operatorname{evac}(\mathrm{Q}(\operatorname{gw}(T)))^{t}$ is $\operatorname{set}(\alpha)$. Thus we have established that $\operatorname{rect}(\operatorname{stan}(T))$ has shape $\operatorname{sort}(\alpha)$ and descent composition $\alpha$. Lemma 5.2 proves the proposition.

This also completes the proof of our main theorem. A remarkable aspect of Proposition 5.3 is that the symmetric group action on LR tableaux via crystal reflection operators translates to the usual permutation action on the parts of the shape underlying the rectification, after applying the $\rho$ map.

5.1. Revisiting the LR RUles in [6]. To conclude this article, we briefly describe an equivalent interpretation of our central result in the language of box-adding operators on compositions. By Proposition 4.6, we know that elements of $\operatorname{LRT}^{\sigma}(\lambda, \mu, \nu)$ may be constructed by computing $\lambda / \mu$-compatible words $w$ satisfying $\operatorname{colform}(w)=\left(w_{0} \sigma w_{0}\right)$. $\nu^{r}$, where $w_{0}$ is the longest word in $\mathfrak{S}_{\ell(\nu)}$. Equivalently, these words are exactly the growth words of standardizations of tableaux in $\operatorname{LRT}^{\sigma}(\lambda, \mu, \nu)$.

Thus, they may be identified with certain saturated chains in Young's lattice from $\mu$ to $\lambda$. By applying the map $\rho_{\beta}$ where sort $(\beta)=\mu$, these chains may be interpreted as chains in $\mathcal{L}_{c}$ from $\beta$ to certain compositions $\gamma$ that satisfy $\operatorname{sort}(\gamma)=\lambda$. Fixing a $\gamma$ and counting these chains allows us to compute $C_{\alpha \beta}^{\gamma}$ where $\alpha=\sigma \cdot \nu$. 
We make the preceding discussion precise by phrasing the result in the language of box-adding operators on compositions introduced in [35] following the seminal work of Fomin [9]. Given $i \geqslant 1$ and a composition $\alpha$ that has at least one part equaling $i-1$, we define $\mathfrak{t}_{i}(\alpha)$ to be the unique composition covering $\alpha$ in $\mathcal{L}_{c}$ where the new box occurs in the $i$-th column. Given a word $w=w_{1} \cdots w_{n}$, define $\mathfrak{t}_{w}:=\mathfrak{t}_{w_{1}} \cdots \mathfrak{t}_{w_{n}}$. We have the following corollary.

Corollary 5.4. Let $\alpha, \beta$ and $\gamma$ be compositions such that $|\beta|+|\alpha|=|\gamma|$. Let $(\lambda, \mu, \nu)$ be the triple $(\operatorname{sort}(\gamma), \operatorname{sort}(\beta), \operatorname{sort}(\alpha))$. If $\sigma \in \mathfrak{S}_{\ell(\nu)}$ satisfies $\alpha=\sigma \cdot \nu$, then

$$
C_{\alpha \beta}^{\gamma}=\left|\left\{w \in \operatorname{Frank}\left(\lambda, \mu,\left(w_{0} \sigma w_{0}\right) \cdot \nu^{r}\right) \mid \mathfrak{t}_{w}(\beta)=\gamma\right\}\right| .
$$

The cases in Corollary 5.4 where $\sigma$ is either the identity or the longest permutation in $\mathfrak{S}_{\ell(\nu)}$ correspond to the left and right LR rules of [6]. We reiterate here that the two LR rules in [6] were proved by different approaches. Thus Corollary 5.4 generalizes these rules by providing a uniform proof that works in all cases.

Acknowledgements. We are very grateful to Sami Assaf for suggesting that we study noncommutative LR coefficients from a crystal perspective and for numerous helpful discussions. We are thankful to Sara Billey and Steph van Willigenburg for various discussions. Finally we are grateful to the anonymous referees for their careful reading and for their very pertinent suggestions to improve exposition.

\section{REFERENCES}

[1] Per Alexandersson, Non-symmetric Macdonald polynomials and Demazure-Lusztig operators, https://arxiv.org/abs/1602.05153, 2016.

[2] Edward E. Allen, Joshua Hallam, and Sarah Mason, Dual immaculate quasisymmetric functions expand positively into Young quasisymmetric Schur functions, J. Combin. Theory Ser. A 157 (2018), 70-108.

[3] Sami Assaf and Dominic Searles, Kohnert tableaux and a lifting of quasi-Schur functions, J. Combin. Theory Ser. A 156 (2018), 85-118.

[4] Chris Berg, Nantel Bergeron, Franco Saliola, Luis Serrano, and Mike Zabrocki, A lift of the Schur and Hall-Littlewood bases to non-commutative symmetric functions, Canad. J. Math. 66 (2014), no. 3, 525-565.

[5] Christine Bessenrodt, Kurt Luoto, and Stephanie van Willigenburg, Skew quasisymmetric Schur functions and noncommutative Schur functions, Adv. Math. 226 (2011), no. 5, 4492-4532.

[6] Christine Bessenrodt, Vasu Tewari, and Stephanie van Willigenburg, Littlewood-Richardson rules for symmetric skew quasisymmetric Schur functions, J. Combin. Theory Ser. A 137 (2016), 179-206.

[7] Anders Skovsted Buch, The saturation conjecture (after A. Knutson and T. Tao), Enseign. Math. (2) 46 (2000), no. 1-2, 43-60, With an appendix by William Fulton.

[8] Daniel Bump and Anne Schilling, Crystal bases. Representations and combinatorics, World Scientific Publishing Co. Pte. Ltd., Hackensack, NJ, 2017.

[9] Sergey Fomin, Schur operators and Knuth correspondences, J. Combin. Theory Ser. A 72 (1995), no. 2, 277-292.

[10] Sergey Fomin and Curtis Greene, A Littlewood-Richardson miscellany, European J. Combin. 14 (1993), no. 3, 191-212.

[11] William Fulton, Young tableaux. With applications to representation theory and geometry, London Mathematical Society Student Texts, vol. 35, Cambridge University Press, Cambridge, 1997.

[12] Israel M. Gel'fand, Daniel Krob, Alain Lascoux, Bernard Leclerc, Vladimir S. Retakh, and JeanYves Thibon, Noncommutative symmetric functions, Adv. Math. 112 (1995), no. 2, 218-348.

[13] Ira M. Gessel, Multipartite P-partitions and inner products of skew Schur functions, in Combinatorics and algebra (Boulder, Colo., 1983), Contemp. Math., vol. 34, Amer. Math. Soc., Providence, RI, 1984, pp. 289-317.

[14] James Haglund, Kurt Luoto, Sarah Mason, and Stephanie van Willigenburg, Quasisymmetric Schur functions, J. Combin. Theory Ser. A 118 (2011), no. 2, 463-490.

[15] Allen Knutson and Terence Tao, The honeycomb model of $\mathrm{GL}_{n}(\mathbf{C})$ tensor products. I. Proof of the saturation conjecture, J. Amer. Math. Soc. 12 (1999), no. 4, 1055-1090. 
[16] Thomas Lam, Aaron Lauve, and Frank Sottile, Skew Littlewood-Richardson rules from Hopf algebras, Int. Math. Res. Not. IMRN (2011), no. 6, 1205-1219.

[17] Alain Lascoux and Marcel-Paul Schützenberger, Le monö̈de plaxique, in Noncommutative structures in algebra and geometric combinatorics (Naples, 1978), Quad. "Ricerca Sci.", vol. 109, CNR, Rome, 1981, pp. 129-156.

[18] Keys E standard bases, in Invariant theory and tableaux (Minneapolis, MN, 1988), IMA Vol. Math. Appl., vol. 19, Springer, New York, 1990, pp. 125-144.

[19] Dudley E. Littlewood and Archibald R. Richardson, Group characters and algebra, Phil. Trans. A 233 (1934), 99-141.

[20] Ricky Ini Liu, An algorithmic Littlewood-Richardson rule, J. Algebraic Combin. 31 (2010), no. 2, 253-266.

[21] M. Lothaire, Algebraic combinatorics on words, Encyclopedia of Mathematics and its Applications, vol. 90, Cambridge University Press, Cambridge, 2002.

[22] Kurt Luoto, Stefan Mykytiuk, and Stephanie van Willigenburg, An introduction to quasisymmetric Schur functions. Hopf algebras, quasisymmetric functions, and Young composition tableaux, SpringerBriefs in Mathematics, Springer, New York, 2013.

[23] Claudia Malvenuto and Christophe Reutenauer, Duality between quasi-symmetric functions and the Solomon descent algebra, J. Algebra 177 (1995), no. 3, 967-982.

[24] Sarah Mason, An explicit construction of type A Demazure atoms, J. Algebraic Combin. 29 (2009), no. 3, 295-313.

[25] Cara Monical, Oliver Pechenik, and Dominic Searles, K-theoretic polynomials, Sém. Lothar. Combin. 82B (2020), Paper no. 10 (12 pages).

[26] Igor Pak and Ernesto Vallejo, Combinatorics and geometry of Littlewood-Richardson cones, European J. Combin. 26 (2005), no. 6, 995-1008.

[27] Victor Reiner and Mark Shimozono, Key polynomials and a flagged Littlewood-Richardson rule, J. Combin. Theory Ser. A 70 (1995), no. 1, 107-143.

[28] , Plactification, J. Algebraic Combin. 4 (1995), no. 4, 331-351.

[29] Jeffrey B. Remmel and Mark Shimozono, A simple proof of the Littlewood-Richardson rule and applications, Discrete Math. 193 (1998), no. 1-3, 257-266, Selected papers in honor of Adriano Garsia (Taormina, 1994).

[30] Bruce E. Sagan, The symmetric group. Representations, combinatorial algorithms, and symmetric functions, second ed., Graduate Texts in Mathematics, vol. 203, Springer-Verlag, New York, 2001

[31] Marcel-Paul Schützenberger, La correspondance de Robinson, in Combinatoire et représentation du groupe symétrique (Actes Table Ronde CNRS, Univ. Louis-Pasteur Strasbourg, Strasbourg, 1976), Lecture Notes in Math., vol. 579, Springer-Verlag, Berlin-New York, 1977, pp. 59-113.

[32] Dominic Searles, Polynomial bases: positivity and Schur multiplication, Trans. Amer. Math. Soc. 373 (2020), no. 2, 819-847.

[33] Richard P. Stanley, Enumerative combinatorics. Vol. 2, Cambridge Studies in Advanced Mathematics, vol. 62, Cambridge University Press, Cambridge, 1999, With a foreword by Gian-Carlo Rota and appendix 1 by Sergey Fomin.

[34] Vasu Tewari, Backward jeu de taquin slides for composition tableaux and a noncommutative Pieri rule, Electron. J. Combin. 22 (2015), no. 1, Paper no. 1.42 (50 pages).

[35] _ A Murnaghan-Nakayama rule for noncommutative Schur functions, European J. Combin. 58 (2016), 118-143.

[36] Glânffrwd P. Thomas, On Schensted's construction and the multiplication of Schur functions, Adv. in Math. 30 (1978), no. 1, 8-32.

[37] Ravi Vakil, A geometric Littlewood-Richardson rule, Ann. of Math. (2) 164 (2006), no. 2, 371421, Appendix A written with A. Knutson.

[38] Marc A. A. van Leeuwen, The Littlewood-Richardson rule, and related combinatorics, in Interaction of combinatorics and representation theory, MSJ Mem., vol. 11, Math. Soc. Japan, Tokyo, 2001, pp. 95-145.

Edward Richmond, Oklahoma State University, Department of Mathematics, Stillwater, OK 74708, USA

E-mail : edward.richmond@okstate.edu

VASU TEwari, University of Pennsylvania, Department of Mathematics, Philadelphia, PA 19104, USA

E-mail : vvtewari@math.upenn.edu 